\title{
Regulation of Leukocyte Recruitment to the Spleen and Peritoneal Cavity during Pristane-Induced Inflammation
}

\author{
Yu Li, ${ }^{1}$ Junping Wu, ${ }^{2,3}$ Long Xu, ${ }^{2}$ Qi Wu, ${ }^{2}$ Zhen Wan, ${ }^{1} \mathrm{Li} \mathrm{Li}^{3}{ }^{3}$ Hongzhi Yu, ${ }^{3}$ Xue Li, ${ }^{1} \mathrm{Kuan} \mathrm{Li}{ }^{1}$ \\ Qiuyang Zhang, ${ }^{1}$ Zhili Hou, ${ }^{4}$ Xin Sun, ${ }^{1,2}$ and Huaiyong Chen $^{1}$ \\ ${ }^{1}$ Department of Basic Medicine, Haihe Clinical College of Tianjin Medical University, Tianjin, China \\ ${ }^{2}$ Key Research Laboratory for Infectious Disease Prevention for State Administration of Traditional Chinese Medicine, Tianjin \\ Institute of Respiratory Diseases, Tianjin Haihe Hospital, Tianjin 300350, China \\ ${ }^{3}$ Department of Respiratory Medicine, Tianjin Haihe Hospital, Tianjin 300350, China \\ ${ }^{4}$ Department of Tuberculosis, Tianjin Haihe Hospital, Tianjin 300350, China
}

Correspondence should be addressed to Xin Sun; 13302031999@126.com and Huaiyong Chen; huaiyong.chen@foxmail.com

Received 16 May 2017; Revised 20 July 2017; Accepted 27 August 2017; Published 19 October 2017

Academic Editor: Elzbieta Kolaczkowska

Copyright (c) $2017 \mathrm{Yu} \mathrm{Li}$ et al. This is an open access article distributed under the Creative Commons Attribution License, which permits unrestricted use, distribution, and reproduction in any medium, provided the original work is properly cited.

\begin{abstract}
Chronic inflammation is associated with an increased number of leukocytes in the spleen, which are then redirected to the site of inflammation. However, it remains unknown how leukocyte recruitment is regulated. Herein, chronic inflammation was induced by intraperitoneal injection of pristane into mice. Leukocytes in the spleen or in the peritoneal cavity were quantified by flow cytometry. We found that the loss of IL-6 decreased macrophage recruitment to the spleen and the peritoneal cavity during pristane-induced inflammation. The loss of TNF $\alpha$ delayed the recruitment of neutrophils and macrophages to the spleen and inhibited the recruitment of neutrophils, macrophages, $\mathrm{B}$ cells, and $\mathrm{T}$ cells. The recruitment of neutrophils and macrophages into the spleen or peritoneal cavity was largely inhibited in the absence of LT $\alpha$. The loss of TNF $\alpha$ receptor $1 / 2$ resulted in reduced recruitment of neutrophils, macrophages, and dendritic cells into the spleen, but only neutrophil recruitment was inhibited in the peritoneal cavity. Similarly, a lack of B cells significantly impeded the recruitment of neutrophils, macrophages, and dendritic cells to the spleen. However, only macrophage recruitment was inhibited in the absence of $\mathrm{T}$ cells in the spleen. These data provide insight into the development of chronic inflammation induced by noninfectious substances.
\end{abstract}

\section{Introduction}

Noninfectious inflammation can be induced by persistent indigestible substances. In many studies, a hydrocarbon oil pristane is often injected intraperitoneally to model noninfectious inflammation. Pristane administration induces macrophage activation [1, 2]. Depending on the genetic background of the model, pristane injection can trigger a local inflammatory response (lipogranuloma), erosive arthritis that resembles rheumatoid arthritis, and systemic lupus erythematosus, followed by autoantibody formation and many clinical manifestations [3-5]. Previous studies from our group and other labs have demonstrated that oil granulomas represent the major pathology in response to pristane injections in C57BL/6 mice [2,3]. Pristane-induced chronic inflammation has been characterized by the continuous recruitment of leukocytes, including lymphocytes, neutrophils, and macrophages, to the peritoneal cavity and the spleen [6-8].

We and others have discovered factors that control the recruitment of inflammatory leukocytes to the peritoneal mesentery in response to pristane $[9,10]$. Cytokines are known to regulate the migration of neutrophils and macrophages during inflammation. Tumor necrosis factor alpha 
(TNF $\alpha$ ) stimulates the expression of monocyte chemoattractant protein 1 (MCP-1) [11]. Mice deficient for TNF $\alpha$ develop defective oil granulomas with reduced recruitment of macrophages and neutrophils [10]. Interleukin-6 (IL-6) seems to regulate both plasmacytoma development in $\mathrm{BALB} / \mathrm{c}$ mice and oil granuloma formation in C57BL/6 mice during pristane-induced inflammation $[10,12]$. Lymphotoxin alpha $(\mathrm{LT} \alpha)$ and $\mathrm{TNF} \alpha$ were shown to induce the expression of homing chemokines in $\mathrm{B}$ and $\mathrm{T}$ cell areas of the spleen [13]. LT $\alpha$ is also required for the recruitment of dendritic cells, neutrophils, and macrophages to the mesentery in response to pristane [10]. Beyond that, LT $\alpha$ also maintains the structure of the mature marginal sinus (MS) in the postnatal spleen [14]. In addition to lymphocytes, dendritic cells can also produce LT $\alpha$ [15]. TNF $\alpha$ plays an important role in the formation of primary $\mathrm{B}$ cell follicles and follicular dendritic cells [16]. TNF $\alpha$ is mainly secreted by primitive neutrophils and participates in the inflammatory response involved in rheumatoid arthritis and inflammatory bowel disease [17].

As the two major cell types in the spleen, B cells and T cells produce cytokines and chemokines [18]. The migration of inflammatory leukocytes, including dendritic cells, neutrophils, and macrophages, to the peritoneal mesentery has been shown to be promoted in $\mathrm{LAT}^{-/-}$(lack mature $\mathrm{T}$ cells) mice but inhibited in $\mu \mathrm{MT}$ (lack mature B cells) mice during the pristane-induced immune response [10]. Thus, leukocyte migration to inflammatory sites in response to pristane has been well characterized. Our previous data demonstrated that lymphocytes and their produced cytokines including TNF $\alpha, \mathrm{LT} \alpha$, and IL- 6 play an important role in such process. We hypothesized that these factors may also be critical for the recruitment of leukocytes to the spleen during pristaneinduced inflammation.

In the present study, chronic inflammation was induced in $\mathrm{C} 57 \mathrm{BL} / 6 \mathrm{~J}$ mice by intraperitoneal injection of pristane to determine whether or not B cells, T cells, IL-6, TNF $\alpha$, and LT $\alpha$ were involved in pristane-induced inflammation via the regulation of dendritic cell, neutrophil, and macrophage recruitment to the spleen. Using flow cytometry to quantitatively analyze the number of leukocytes in the spleen, we observed that the recruitment of dendritic cells, neutrophils, and macrophage to the spleen followed different regulatory patterns.

\section{Materials and Methods}

2.1. Mouse Strains and Pristane Administration. $\mu M T$ mice [19], $T N F \alpha^{-/-}$mice, $L T \alpha^{-/-}$mice, $I L-\sigma^{-1-}$ mice, TNFR $1 / 2^{-/-}$ mice, and $L A T^{-1-}$ mice were purchased from the Jackson Laboratories (Bar Harbor, ME, USA). In these studies, all animals were housed and cared for in a specific pathogenfree facility with sterile bedding, water, and food. Therefore, any effects of incidental antigen exposure on inflammation were minimized. All animal experiments were approved by the Animal Care and Use Committee at Haihe Clinical College of Tianjin Medical University. To induce granuloma inflammation, adult mice ( $8-10$ weeks old) received a single intraperitoneal (i.p.) injection of pristane $(100$ or $300 \mu \mathrm{L}$;
$2.8 \times 10^{-4}$ or $8.3 \times 10^{-4} \mathrm{~mol}$, resp., $\geq 95 \%$ purity, SigmaAldrich, St. Louis, MO, USA). Age-matched, untreated mice were used as controls. Three to six WT mice or knockout mice were sacrificed at each time point after pristane.

2.2. Preparation of Splenic Cells and Resident Peritoneal Cells. After anesthesia with i.p. injection of pentobarbital sodium $(100 \mathrm{mg} / \mathrm{kg})$, cervical dislocation was then performed to sacrifice mice at various time points following an injection of pristane. The abdominal cavity was opened, and the complete spleen was removed from C57BL/6J mice or the specific gene knockout mice. Spleens were minced in RPMI-1640 media (Gibco) at room temperature $\left(21^{\circ} \mathrm{C}\right)$ to prepare single-cell suspensions. The resulting cell suspension was removed and filtered through a fine nylon mesh (Denville Scientific Inc., Metuchen, NJ, USA). Resident peritoneal cavity (PC) cells were collected by lavage with $10 \mathrm{~mL}$ of ice-cold RPMI-1640 supplemented with 5\% FBS. After centrifugation, the cell pellets were harvested and counted with a cytometer.

2.3. Flow Cytometry. Suspensions of splenic cells or peritoneal cells prepared above were incubated with ammonium chloride buffer for $1 \mathrm{~min}$ on ice to lyse red blood cells (RBCs) before immunolabeling. Nucleated cells were labeled with antibodies specific for B220 (PE-Cy7), TCR $\beta$ (APC), CD11c (PE), Gr-1 (FITC), and CD11b (APC-Cy7) for $20 \mathrm{~min}$ on ice. To identify and exclude dead cells, DAPI (7-AAD Viability Staining Solution, eBioscience) was used. Flow cytometric data were analyzed with FlowJo software (Tree Star, Ashland, OR, USA). Labeled cells were analyzed in a FACSVantage with DIVA option. The absolute number of each cell type in each sample was determined by multiplying the total number of cells with the percentage of each cell type in the same sample.

2.4. Quantitative PCR. Mice were anesthetized and sacrificed as mentioned above. The peritoneal mesentery was harvested and minced. Total RNA was extracted from the tissue using TRIzol reagent (Invitrogen, Carlsbad, CA, USA). Messenger RNA was reverse transcribed with oligo (dT) primer for $1 \mathrm{~h}$ at $50^{\circ} \mathrm{C}$. Quantitative PCR was performed in an iCycler Thermal Cycler with SYBR ${ }^{\circledR}$ Green PCR core reagents (Applied Biosystems, Foster City, CA) and primers for specific genes. Amplification conditions were as follows: denaturation at $94^{\circ} \mathrm{C}$ for $10 \mathrm{~min}$ and amplification at $94^{\circ} \mathrm{C}$ for $15 \mathrm{~s}$ and $60^{\circ} \mathrm{C}$ for $45 \mathrm{~s}$, repeated for 40 cycles. Primers included were as follows: $\beta$-actin, forward 5 -AGCCATGTACGTAGCCA TCC-3 and reverse 5 '-CTCTCAGC TGTGGTGGTGAA-3'; TNF $\alpha$, forward $5^{\prime}$-TCCCCAAAGGGATGAGAAGTTC-3' and reverse 5'-GGGAGTAGACAAGGTACAAC-3'; IL- $1 \beta$, forward 5'-GGTACATCAGCACCTCACAA-3' and reverse 5'-TTAGAAACAGTCCAGCCCATAC-3'; and IL-6, forward $5^{\prime}$-CCCAACAGACCTGTCTATACC- $3^{\prime}$ and reverse $5^{\prime}$ CAGCTTATCTGTTAGGAGAGC-3'. The relative mRNA levels for the indicated target genes were calculated by the comparative $\mathrm{Ct}$ (threshold cycle) method and normalized to $\beta$-actin levels in the same sample. 
2.5. Statistical Analyses. Data were collected from three or more replicate samples in each independent experiment. Differences between paired groups were analyzed using Student's $t$-test; $p$ values $\leq 0.05$ were considered significant.

\section{Results}

3.1. Dose-Dependent Recruitment of Leukocytes to the Spleen. To investigate splenic leukocyte responses to pristane, C57BL/6J mice were injected intraperitoneally with a single dose of 100 or $300 \mu \mathrm{L}$ of pristane. Mice were then sacrificed to prepare single-cell suspensions at various time points. Whole splenic cells were analyzed using flow cytometry after staining with antibodies specific for B220, TCR $\beta$, CD11c, $\mathrm{CD} 11 \mathrm{~b}$, and $\mathrm{Gr}-1 . \mathrm{CD} 11 \mathrm{c}^{+}$dendritic cells, CD $11 \mathrm{c}^{-} / \mathrm{B} 220^{+} \mathrm{B}$ cells, CD $11 \mathrm{c}^{-} / \mathrm{TCR} \beta^{+} \mathrm{T}$ cells, CD $11 \mathrm{c}^{-} / \mathrm{CD} 11 \mathrm{~b}^{+} / \mathrm{Gr}-1^{\text {hi }}$ neu-

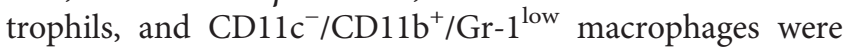
fractionated and counted (Figure 1(a)). The number of $\mathrm{B}$ cells, $T$ cells, and dendritic cells in the spleen was unchanged during pristane treatment at both doses (Figure 1(b)). However, the responses of neutrophils and macrophages to pristane were dose dependent (Figure 1(b)). In the presence of low-dose pristane, the number of both neutrophils and macrophages was augmented at week three, which then decreased and returned to their basal levels at 11 weeks posttreatment (Figure 1(b)). In contrast, the injection of highdose pristane resulted in the recruitment of significantly more neutrophils and macrophages to the spleen throughout the treatment period (Figure 1(b)). Therefore, $300 \mu \mathrm{L}$ of pristane was chosen for the subsequent experiments.

We also collected mesenteric tissues from the peritoneal cavity and observed that the mRNA of the inflammatory cytokine TNF $\alpha$ was rapidly elevated at week three after treatment with $300 \mu \mathrm{L}$ of pristane and then gradually returned to the basal level by week 11 (Figure 1(c)). Likewise, the expression of IL- $1 \beta$ and IL- 6 in the peritoneal mesentery followed a similar trend during pristane-induced inflammation (Figure 1(c)). These data suggested that TNF $\alpha$ and IL-6 are associated with pristane-induced inflammation and may play a role in the recruitment of leukocytes to the spleen.

3.2. IL-6 Promotes Recruitment of Macrophages to the Spleen in Response to Pristane. To investigate a possible role IL-6 in leukocyte recruitment to the spleen during pristaneinduced inflammation, we injected IL- $6^{-/-}$mice with $300 \mu \mathrm{L}$ of pristane intraperitoneally. At the steady state, the total number of splenic cells decreased slightly in IL- $6^{-/-}$mice as compared to the total number of splenic cells in wild-type (WT) mice (Figure 2(a)), which may be due to the significant reduction in the number of $B$ cells and $T$ cells (Figure $2(b)$ ). The number of $\mathrm{B}$ cells and $\mathrm{T}$ cells remained attenuated in IL- $6^{-1-}$ mice when compared to the number of $\mathrm{B}$ cells and $\mathrm{T}$ cells in WT mice three weeks after pristane treatment (Figure 2(b)). Pristane treatment promoted the recruitment of both neutrophils and macrophages to the spleen (Figure 2(b)). In the absence of IL-6, the recruitment of neutrophils in response to pristane treatment remained intact (Figure 2(b)). However, the recruitment of macrophages was inhibited in the absence of IL-6 during pristane- induced inflammation (Figure 2(b)). In the peritoneal cavity, pristane injection led to an increased infiltration of cells, which was abolished by IL-6 loss (Figure 2(c)). Neutrophils and macrophages represented the two major mononuclear cell infiltrates in the peritoneal cavity after pristane treatment (Figure 2(d)). IL-6 loss significantly inhibited the infiltration of neutrophils and macrophages into the peritoneal cavity after pristane treatment (Figure 2(d)). These data demonstrated that IL-6 is required for the infiltration of both neutrophils and macrophages into the peritoneal cavity and for the recruitment of only macrophages to the spleen during pristane-induced inflammation.

3.3. LT $\alpha$ Enhanced the Recruitment of Both Neutrophils and Macrophages to the Spleen in Response to Pristane. To determine whether LT $\alpha$ affects recruitment of leukocytes to the spleen, $L \mathrm{~T}^{-/-}$mice were injected with $300 \mu \mathrm{L}$ of pristane. At the steady state, the total number of splenic cells in $L T \alpha^{-/-}$mice was higher than the total number of splenic cells in WT mice (Figure 3(a)), which may be due to the slight increase in B cells and T cells (Figure 3(b)). LT $\alpha$ deficiency had little effect on the number of dendritic cells at the steady state. During pristane-induced inflammation, the recruitment of both neutrophils and macrophages to the spleen was significantly blocked (Figure 3(b)). Likewise, the infiltration of neutrophils and macrophages into the peritoneal cavity was also inhibited in the absence of LT $\alpha$, resulting in a decrease in the number of total cell infiltrates during pristane-induced inflammation (Figures 3(c) and 3(d)). It appeared that the dendritic cells were cleared faster from the peritoneal cavity in the $\mathrm{LT} \alpha^{-1-}$ mice than in the WT mice (Figure 3(d)). This observation suggested that $\mathrm{LT} \alpha$ is critical for the recruitment of both neutrophils and macrophage in the spleen and the peritoneal cavity during pristane-induced inflammation.

3.4. TNF $\alpha$ Is Required for Rapid Mounting of the Inflammatory Response to Pristane in the Spleen. TNF $\alpha^{-1-}$ mice were also used in the present study to examine whether or not $\mathrm{TNF} \alpha$ regulates the inflammatory response to pristane in the spleen. For this, $T N F \alpha^{-1-}$ mouse and WT mouse controls were injected intraperitoneally with $300 \mu \mathrm{L}$ of pristane. TNF $\alpha$ loss had little to no effect on the total number of splenic cells at the steady state or during pristane-induced inflammation (Figure 4(a)). However, dendritic cells seemed to increase in the absence of $\mathrm{TNF} \alpha$ and after pristane treatment (Figure 4(b)). The number of both neutrophils and macrophages in the spleen was significantly lower in $\mathrm{TNF}^{-/-}$mice than the number of neutrophils and macrophages in WT mice at week three but was similar at week five after pristane treatment (Figure 4(b)). In the peritoneal cavity, cell infiltration was largely inhibited (Figure 4(c)). Among all cell types, fewer B cells and T cells infiltrated into the peritoneal cavity after pristane treatment (Figure $4(\mathrm{~d})$ ). Clearance of neutrophils was faster in the absence of TNF $\alpha$, while macrophage recruitment was almost completely blocked (Figure 4(d)). These data suggested that TNF $\alpha$ is required for the rapid response to pristane in the spleen but exhibited a more complicated regulatory role in the immune response to pristane in the peritoneal cavity. 

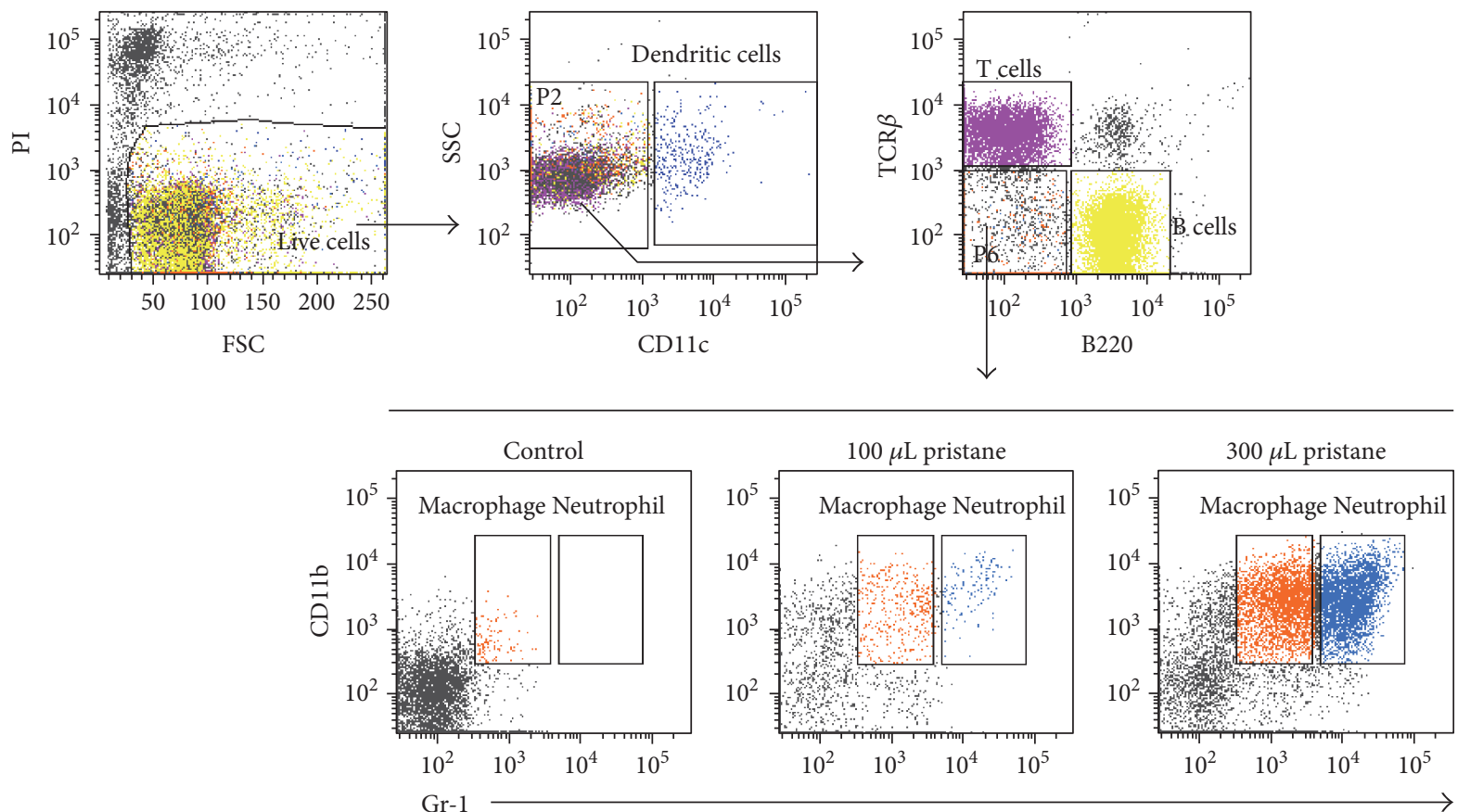

(a)



$\diamond 100 \mu \mathrm{L}$ pristane

$300 \mu \mathrm{L}$ pristane

(b)
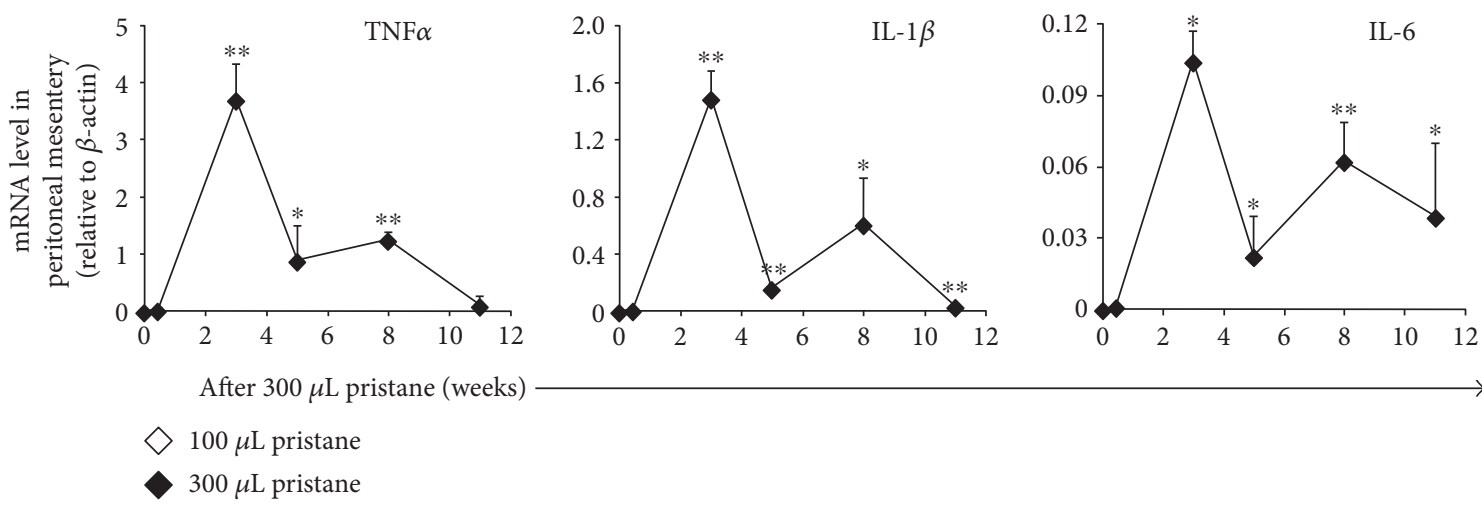

$\diamond 100 \mu \mathrm{L}$ pristane

$\mu \mathrm{L}$ pristane

(c)

FIGURE 1: Leukocyte responses to pristane treatment were dose dependent. Whole splenic cells were analyzed using flow cytometry after staining with antibodies specific for B220, TCR $\beta, \mathrm{CD} 11 \mathrm{c}, \mathrm{CD} 11 \mathrm{~b}$, and Gr-1 (a). The number of B cells (B220 $\left.0^{+}\right)$, T cells (TCR $\left.\beta^{+}\right)$, dendritic cells $\left(\mathrm{CD} 11 \mathrm{c}^{+}\right)$, macrophages $\left(\mathrm{CD} 11 \mathrm{~b}^{+} \mathrm{Gr} 1^{\text {low }}\right)$, or neutrophils $\left(\mathrm{CD} 11 \mathrm{~b}^{+} / \mathrm{Gr} 1^{\text {hi }}\right)$ in the spleen was quantified (b). The difference between $100 \mu \mathrm{L}$ of pristane treatment and $300 \mu \mathrm{L}$ of pristane treatment in C57BL/6J mice is shown. (c) Peritoneal mesentery was collected from $\mathrm{C} 57 \mathrm{BL} / 6$ mice treated with $300 \mu \mathrm{L}$ of pristane and analyzed for the expression of cytokines. The difference between $300 \mu \mathrm{L}$ pristane-treated and nontreated C57BL/6J mice is shown. Data represent two independent experiments. ${ }^{*} p<0.05$ and ${ }^{* *} p<0.01$. 


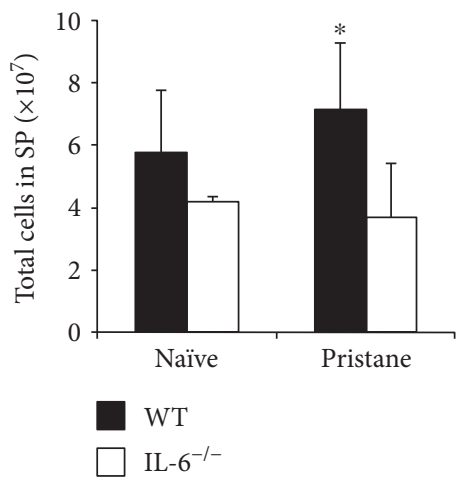

(a)
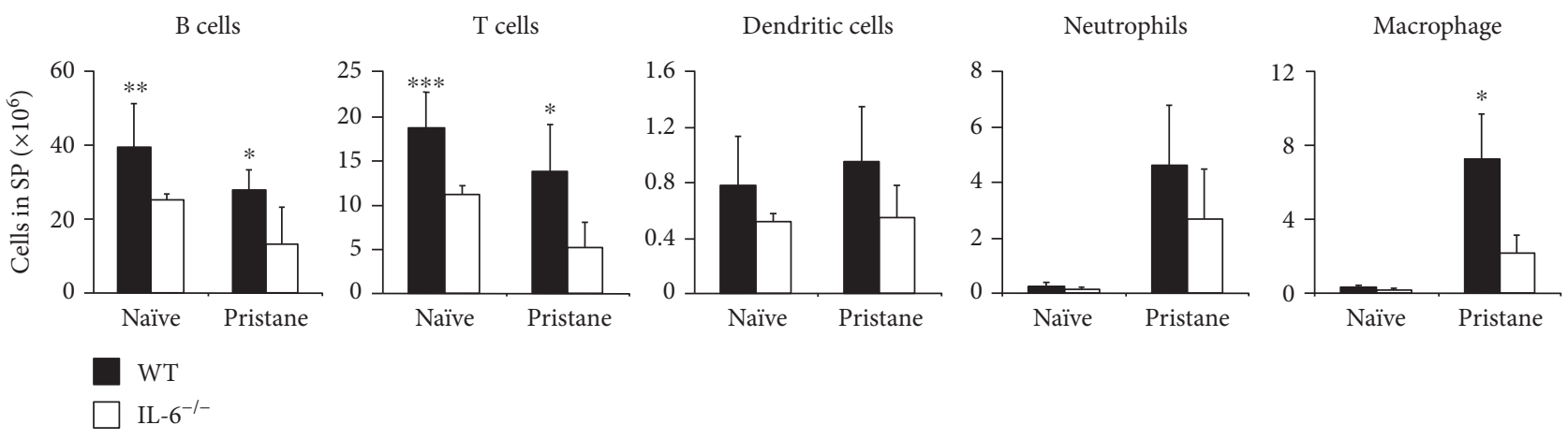

(b)



(c)
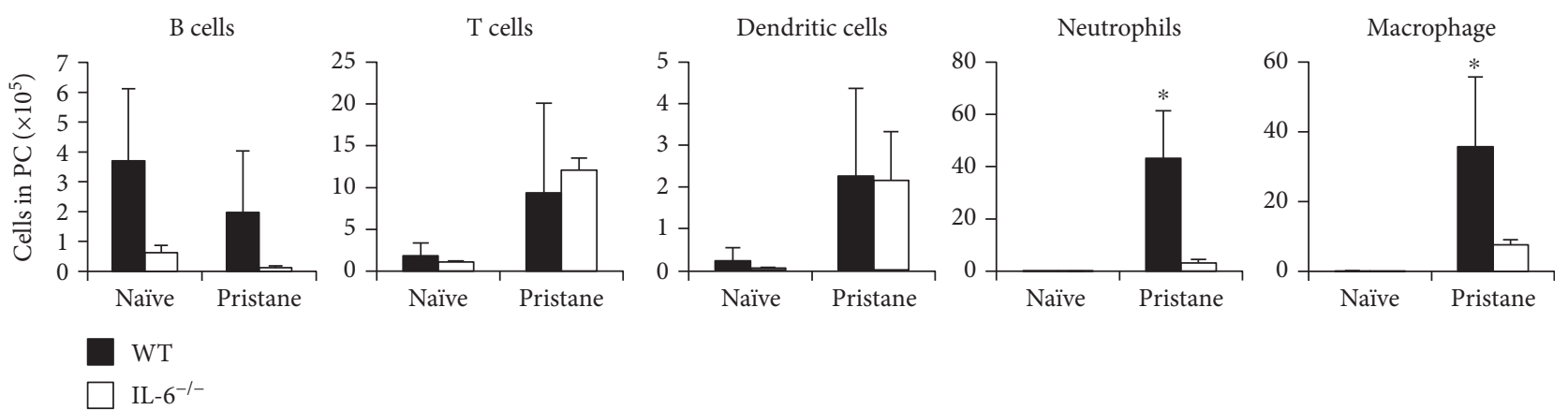

(d)

Figure 2: IL-6 deficiency decreased the recruitment of splenic macrophages in response to pristane treatment. (a, b) IL- $6^{-1-}$ mice and WT mice were injected with $300 \mu \mathrm{L}\left(8.3 \times 10^{-4} \mathrm{~mol}\right)$ of pristane and sacrificed at three weeks thereafter. Single cell suspensions of the spleen (SP) and peritoneal cavity (PC) from pristane-injected WT and $I L-\sigma^{\prime-}$ mice were prepared for cellularity analysis by flow cytometry. The total cell number and the number of each cell type were calculated, respectively. (c, d) The total number of cells or the number of each cell type in the PC was analyzed in the same way. The difference between WT and $I L-\sigma^{\prime-}$ mice is shown. Data represent two independent experiments. ${ }^{*} p<0.05,{ }^{* *} p<0.01$, and ${ }^{* * *} p<0.001$. 


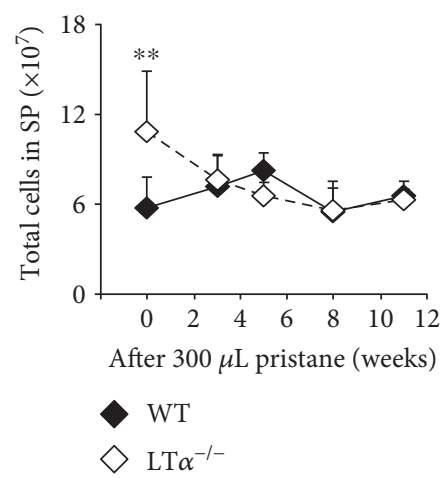

(a)
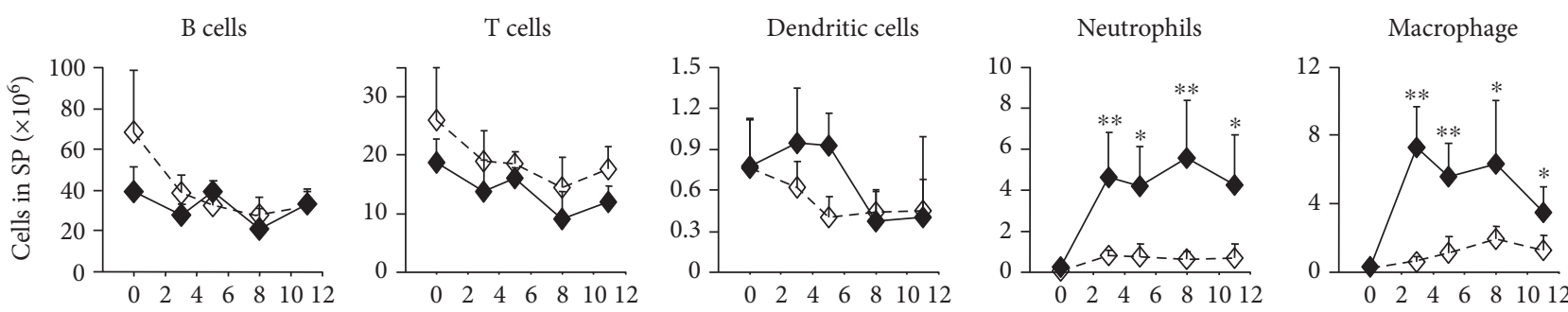

$\diamond \mathrm{WT}$
$\diamond \mathrm{LT}^{-1-}$

(b)

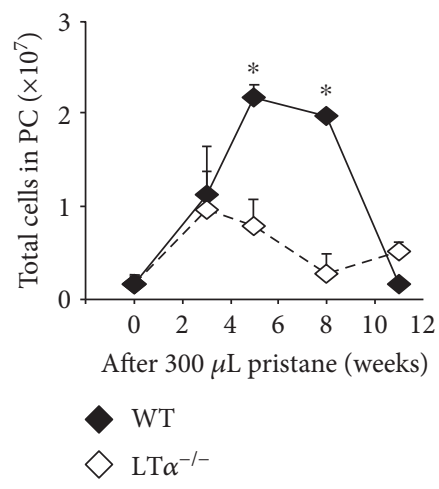

(c)
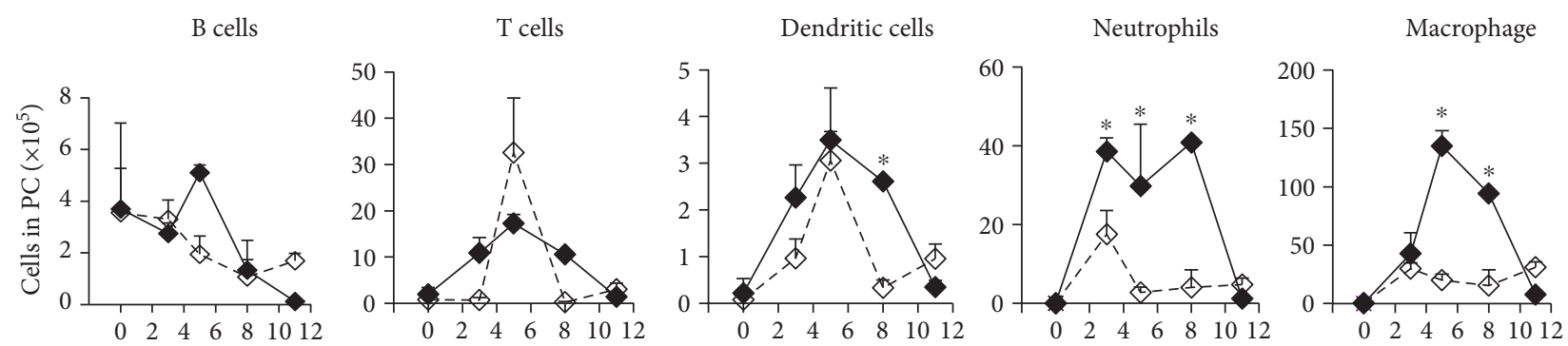

After $300 \mu \mathrm{L}$ pristane (weeks)

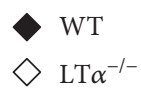

(d)

FIGURE 3: LT $\alpha$ loss inhibited the recruitment of both splenic neutrophils and macrophages in response to pristane treatment. (a, b) $L T \alpha^{-1-}$ mice and WT mice were injected with $300 \mu \mathrm{L}\left(8.3 \times 10^{-4} \mathrm{~mol}\right)$ of pristane and were then sacrificed at the indicated time points. The total number of cells and the number of each cell type were calculated. (c, d) The total number of cells or the number of each cell type in the PC was also analyzed. The difference between WT and $L T \alpha^{-1-}$ mice is shown. Data represent two independent experiments. ${ }^{*} p<0.05$ and ${ }^{* *} p<0.01$. 


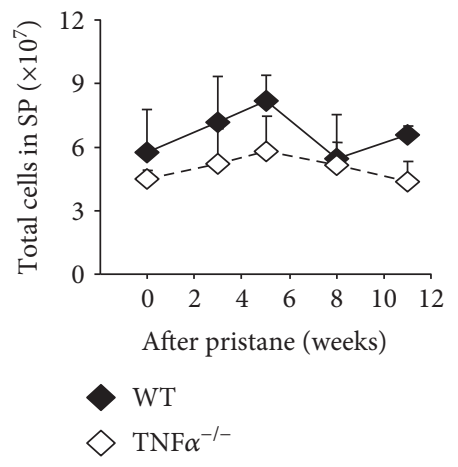

(a)
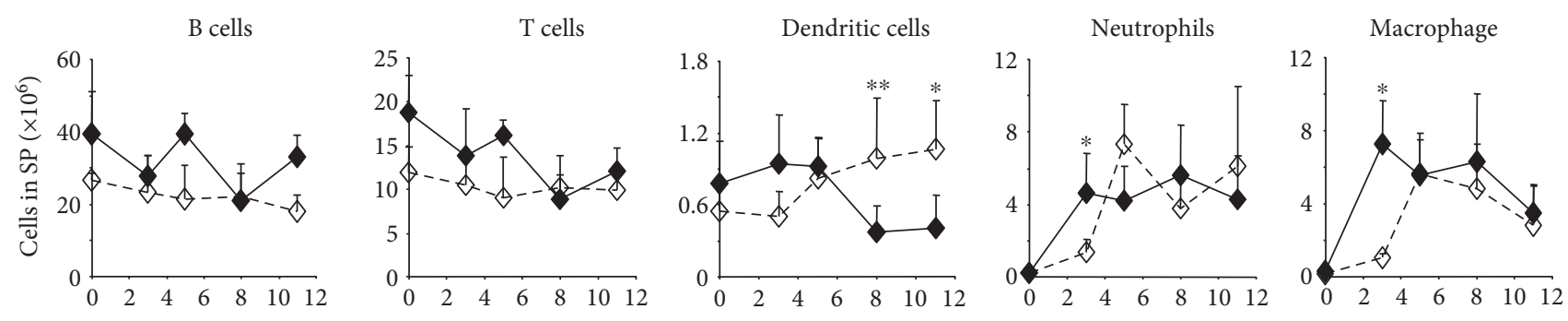

$\checkmark \mathrm{WT}$

$\diamond \mathrm{TNF} \alpha^{-l-}$

(b)

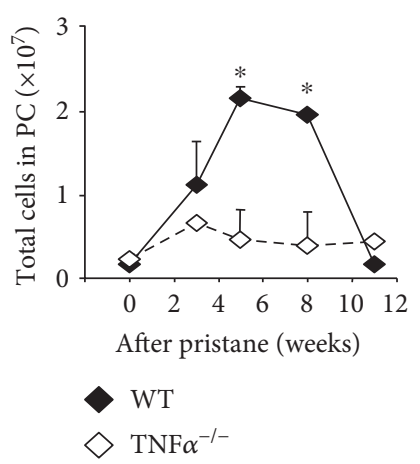

(c)
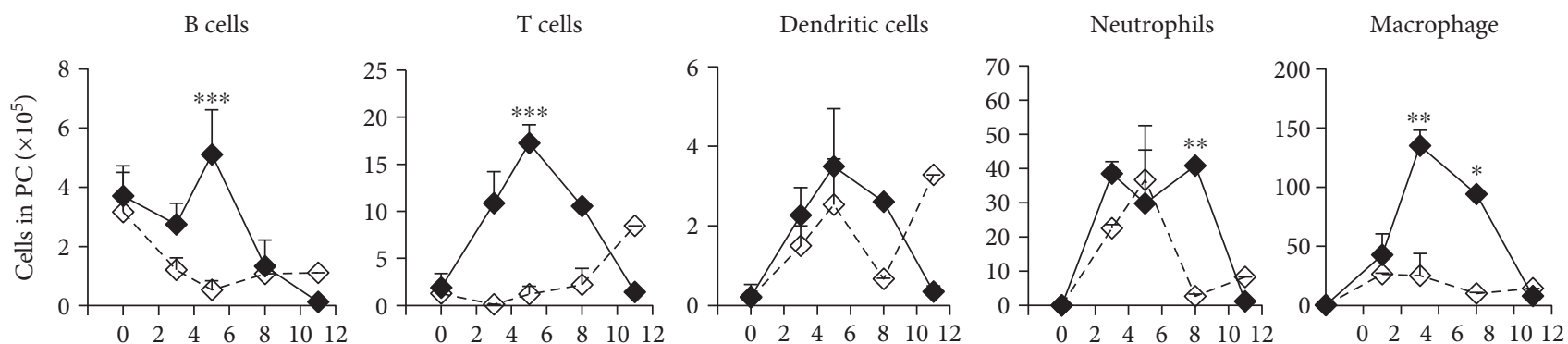

After $300 \mu \mathrm{L}$ pristane (weeks)

- WT

$\diamond \mathrm{TNF} \alpha^{-1-}$

(d)

FIGURE 4: Lack of TNF $\alpha$ delayed the recruitment of splenic neutrophils and macrophages in response to pristane treatment. (a, b) TNF $\alpha^{-1-}$ mice and WT mice were injected with $300 \mu \mathrm{L}\left(8.3 \times 10^{-4} \mathrm{~mol}\right)$ of pristane and were then sacrificed at the indicated time points. The total number of cells and the number of each cell type were calculated. (c, d) The total number of cells or the number of each cell type in the PC was also analyzed. The difference between WT and $T N F \alpha^{-1-}$ mice is shown. Data represent two independent experiments. ${ }^{*} p<0.05$, ${ }^{* *} p<0.01$, and ${ }^{* * *} p<0.001$. 


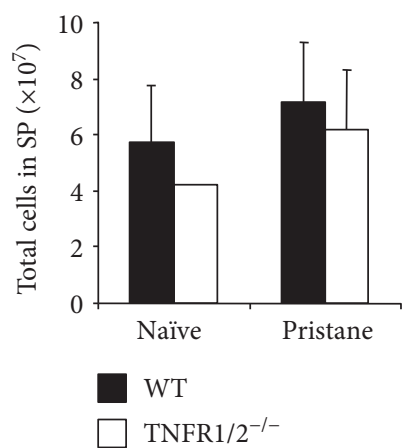

(a)
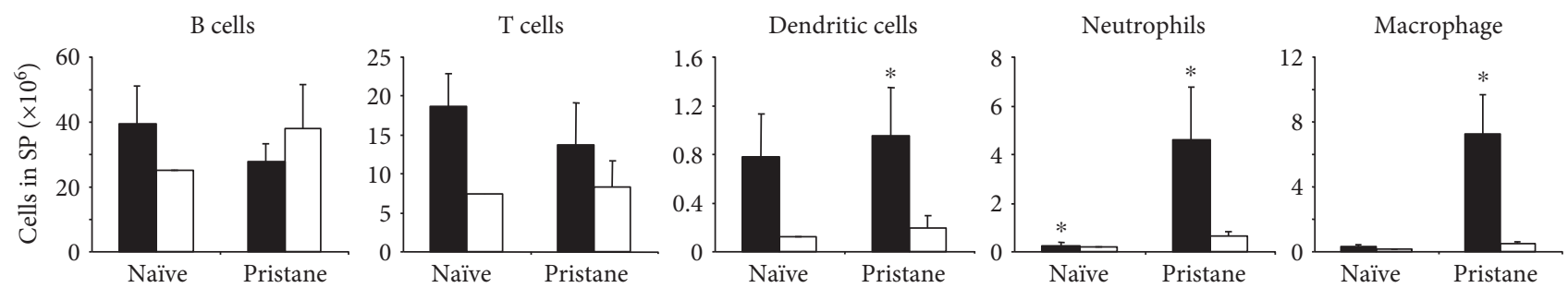

WT

TNFR $1 / 2^{-/-}$

(b)

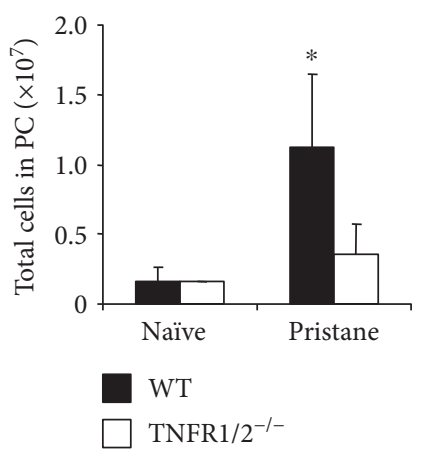

(c)
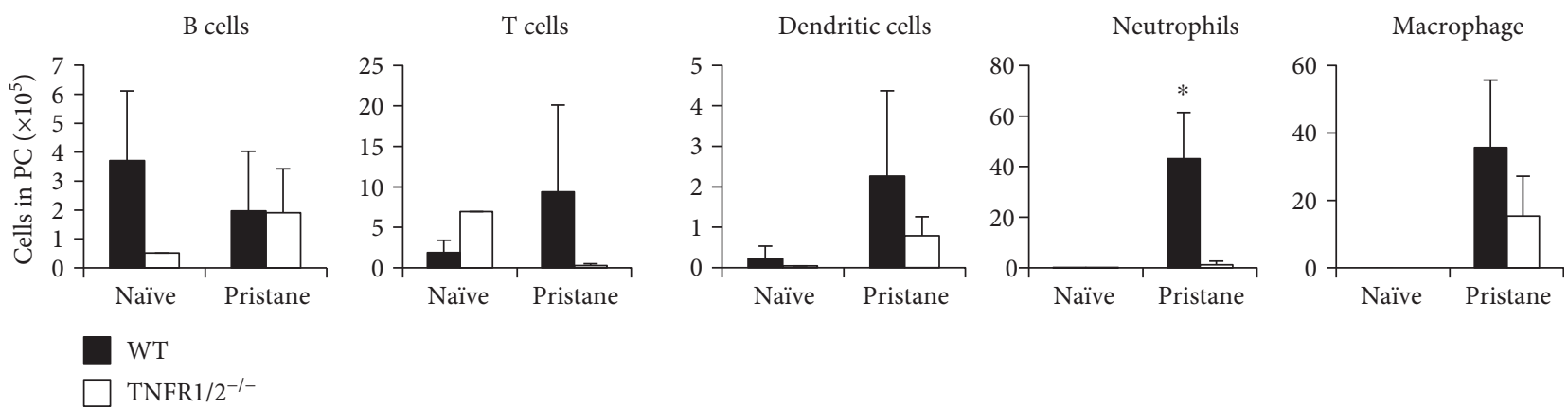

(d)

FIgURE 5: The absence of TNFR1/2 inhibited the recruitment of splenic neutrophils, macrophages, and dendritic cells in response to pristane treatment. (a, b) TNFR1/2 $2^{-1-}$ mice and WT mice were injected with $300 \mu \mathrm{L}\left(8.3 \times 10^{-4} \mathrm{~mol}\right)$ of pristane and sacrificed at three weeks thereafter. Total number of cells and the number of each cell type were calculated. (c, d) Total number of cells and the number of each cell type in the PC were also analyzed. The difference between WT and TNFR $1 / 2^{-1-}$ mice is shown. Data represent two independent experiments. ${ }^{*} p<0.05$.

3.5. TNFR1/2 Promoted Recruitment of Neutrophils and Macrophages to the Spleen. As TNF $\alpha$ was shown to regulate pristane-induced inflammation, we predicted that TNF $\alpha$ receptors may also play a role. Using TNFR $1 / 2^{-/-}$mice to examine this hypothesis, the recruitment of both neutrophils and macrophages to the spleen was largely inhibited in the absence of TNFR1/2, as expected; furthermore, the total number of splenic cells remained unchanged three weeks 


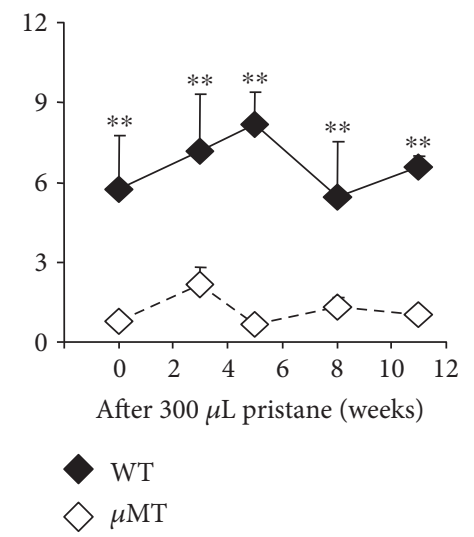

(a)
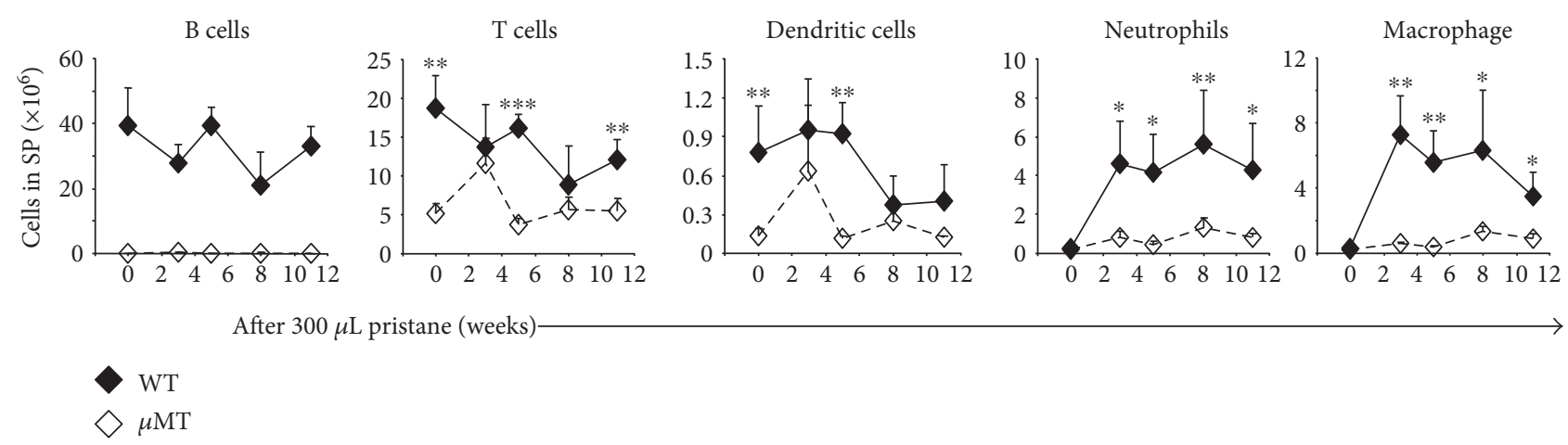

(b)

Figure 6: B cell loss reduced the cellularity of both leukocytes and lymphocytes during pristane-induced splenic inflammation. (a, b) $\mu M T$ mice and WT mice were injected with $300 \mu \mathrm{L}\left(8.3 \times 10^{-4} \mathrm{~mol}\right)$ of pristane and were then sacrificed at the indicated time points. The total number of cells and the number of each cell type were calculated. The difference between WT and $\mu M T$ mice is shown. Data represent two independent experiments. ${ }^{*} p<0.05,{ }^{* *} p<0.01$, and ${ }^{* * *} p<0.001$.

after pristane treatment (Figures 5(a) and 5(b)). Moreover, the dendritic cells in the spleen also appeared to have their recruitment inhibited (Figure 5(b)). In the peritoneal cavity, TNFR1/2 loss resulted in an attenuation of cell infiltration after pristane treatment (Figure 5(c)). Macrophage recruitment to the peritoneal cavity remained intact in response to pristane treatment even in the absence of TNFR1/2, but neutrophil recruitment was almost fully inhibited (Figure 5(d)). These data suggested that TNF $\alpha$ receptors play a more profound role than $\mathrm{TNF} \alpha$ in regulating pristaneinduced inflammation.

3.6. B Cells Promote the Recruitment of Neutrophils and Macrophages to the Spleen. As a major cell type in the spleen, $B$ cells are known to produce inflammatory cytokines, including TNF $\alpha$. Next, we sought to examine whether or not B cells were involved in the pristane-induced inflammatory response by utilizing $\mu M T$ mice, which lack B cells. At the steady state, loss of B cells led to a significant drop in the number of T cells and dendritic cells, as well as the total number of splenic cells (Figures 6(a) and 6(b)). Following pristane-induced inflammation, the number of all cell types analyzed, including $\mathrm{T}$ cells, dendritic cells, neutrophils, and macrophages, was reduced in the absence of $\mathrm{B}$ cells when compared to the number of cell of each type in the WT controls (Figure 6(b)). This finding suggested that B cells are crucial for the recruitment of dendritic cells, neutrophils, and macrophages during the pristane-induced inflammatory response in the spleen.

3.7. T Cells Were Crucial for the Recruitment of Splenic Macrophages in Response to Pristane. We next sought to examine whether or not $\mathrm{T}$ cells, the other major cell type in the spleen, were important in the pristane-induced inflammatory response. To do so, we utilized $L A T^{-1-}$ mice. At the steady state, loss of $\mathrm{T}$ cells led to a significant reduction in the number of $\mathrm{B}$ cells, as well as the resulting reduction in total number of splenic cells (Figures $7(a)$ and $7(b)$ ). Three weeks postpristane injection, only the number of macrophages was reduced in the absence of $\mathrm{T}$ cells, as compared to T cell abundance in the WT controls (Figure 7(b)). This finding suggested that $\mathrm{T}$ cells are important for the recruitment of macrophages during pristane-induced inflammatory response in the spleen.

\section{Discussion}

In the present study, chronic inflammation was modeled by injecting pristane into the peritoneal cavity of mice. The regulation of leukocyte recruitment to the spleen during chronic 


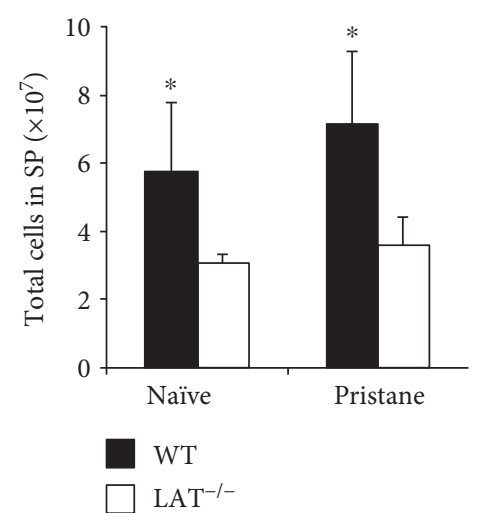

(a)
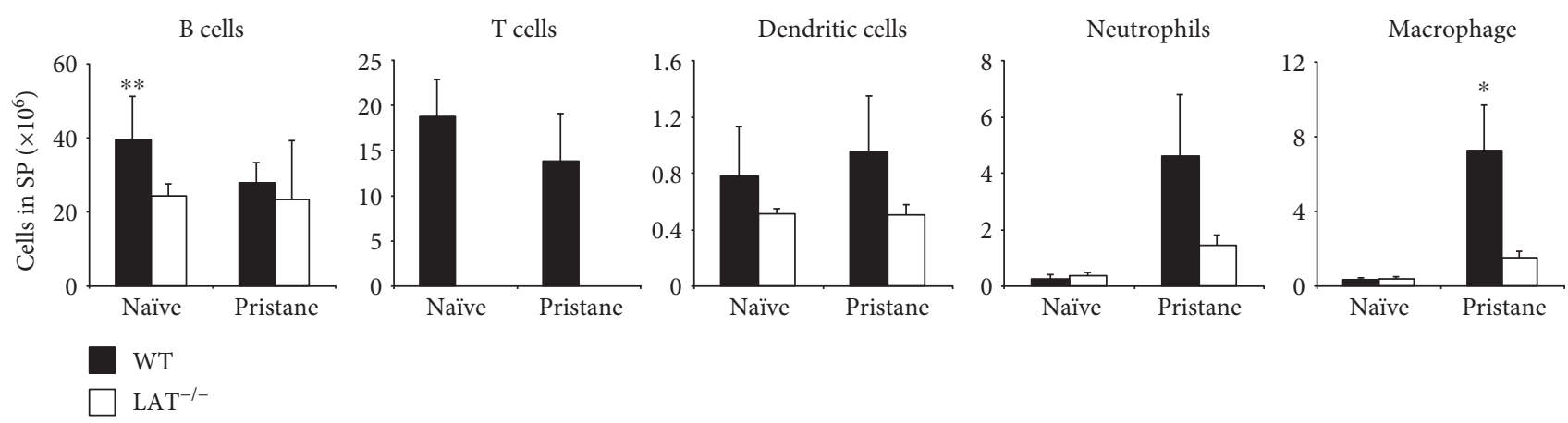

(b)

FIGURE 7: T cell loss inhibits splenic macrophage recruitment during pristane-induced splenic inflammation. (a, b) $L A T^{-1-}$ mice and WT mice were injected with $300 \mu \mathrm{L}\left(8.3 \times 10^{-4} \mathrm{~mol}\right)$ of pristane and sacrificed at three weeks thereafter. Total number of cells and the number of each cell type were calculated. The difference between $\mathrm{WT}$ and $L A T^{-1-}$ mice is shown. Data represent two independent experiments. ${ }^{*} p<0.05$ and ${ }^{* *} p<0.01$.

inflammation was mapped utilizing mice deficient in B cells, $\mathrm{T}$ cells, or genes known to participate in the inflammatory response. Recruitment of macrophages to spleen required splenic T cells during pristane-induced inflammation. B cells were important not only for macrophage recruitment but also for dendritic cell and neutrophil recruitment. Additionally, IL-6 promoted the recruitment of macrophages to the spleen. Moreover, LT $\alpha$ enhanced the recruitment of macrophages and neutrophils. However, TNF $\alpha$ deficiency slowed the recruitment process of neutrophils and macrophages to the spleen and TNFR1/2 promoted the recruitment of macrophages and neutrophils in a similar manner to B cells.

As a major cell type in spleen, B cells play a crucial role in regulating the immune response. $\mathrm{B}$ cells can regulate experimental autoimmune encephalomyelitis (EAE) via the production of interleukin-10 (IL-10) [20]. Induction of B cell-derived IL-10 provides protection against EAE [21]. Furthermore, IL-10 derived from B cells restrains the development of ulcerative colitis by suppressing $\mathrm{T}$ helper $\left(\mathrm{T}_{\mathrm{H}}\right)_{2}$ type immune pathology and reduces the production of $\mathrm{T}_{\mathrm{H}} 2$ cell-mediated IL-4, which can trigger the disease [22, 23]. Although B cells may not act in the same way in different diseases when regulating the immune system, B cell deficiency may provide protection against chronic inflammation, or perhaps the absence of $\mathrm{B}$ cells can alter antigen presentation by dendritic cells and macrophages to $\mathrm{B}$ and $\mathrm{T}$ cells, in turn perturbing the feedback regulation between lymphocytes, dendritic cells, and macrophages. We previously reported that the absence of B cells almost fully inhibited the recruitment of dendritic cells, neutrophils, and macrophages to the peritoneal cavity during pristane-induced chronic inflammation [10]. A similar regulatory pattern was observed in the spleen for the present study, suggesting that B cells also are crucial in mounting a noninfectious inflammatory response. In contrast to $\mathrm{B}$ cells, $\mathrm{T}$ cells play a limited role in pristane-induced inflammation; only macrophage recruitment required $\mathrm{T}$ cells. Pristane-induced arthritis has been proposed to be $\mathrm{CD}^{+} \mathrm{T}$ cell dependent [24].

Differential roles of $\mathrm{B}$ cells and $\mathrm{T}$ cells in regulating pristane-induced inflammation suggested that cytokines may play differential roles in regulating pristane-induced noninfectious chronic inflammation. Our results indicated that TNF $\alpha$ helped quickly mount the inflammatory response to pristane treatment. Lack of $\mathrm{TNF} \alpha$ receptors resulted in a limited response to pristane treatment in the spleen and in the peritoneal cavity. The use of an anti-TNF $\alpha$ antibody or recombinant soluble $\mathrm{TNF} \alpha$ receptors to inactivate $\mathrm{TNF} \alpha$ ameliorated inflammation within mice with collageninduced arthritis [25]. TNF $\alpha$ AU-rich elements (AREs) are targets for p38 and JNK kinase signaling, and deletion of TNF $\alpha$ AREs leads to the overexpression of TNF $\alpha$, further exacerbating chronic inflammatory arthritis and 
inflammatory bowel disease [26]. Unlike $\operatorname{TNF} \alpha$, dendritic cell-derived LT $\alpha$ modulates the production of IgA and cellularity of secondary lymphoid tissue, in turn influencing dendritic cell homeostasis $[15,27,28]$. The absence of LT $\alpha$ signaling blocks $\operatorname{IgE}$ synthesis and disrupts the balance in mouse $\mathrm{CD} 4^{+} \mathrm{T}_{\mathrm{H}}$ cell subsets, as well as in the spleen and lungs of mice lacking $\mathrm{LT} \alpha$ express higher levels of $\mathrm{T}_{\mathrm{H}} 1$-type cytokines [29]. Herein, we demonstrated that a lack of LT $\alpha$ blocks the accumulation of macrophages and neutrophils in the spleen and in the peritoneal cavity during pristane-induced inflammation. LT $\alpha$ and TNF $\alpha$ have been shown to play independent roles in the evolution of the granulomatous response in experimental leprosy because of their distinctive roles in the recruitment of lymphocytes. Unlike TNF $\alpha$ and LT $\alpha$, IL- 6 is only required for macrophage recruitment to the spleen in response to pristane treatment. Therefore, chemokine expression may be regulated differentially in various pathologies associated with the expression of different cytokines. This would need to be clarified in future studies.

Furthermore, cytokines play differential roles in directing leukocyte accumulation to the spleen or to the peritoneal cavity during pristane-induced noninfectious inflammation. IL-6 promotes the recruitment of both neutrophils and macrophages to the peritoneal cavity; but in the spleen, it directs the recruitment of macrophages only. TNF $\alpha$ delays the recruitment of neutrophils and macrophages to the spleen; however, it promotes the clearance of neutrophils and blocks the recruitment of macrophages in the peritoneal cavity. Unlike to IL- 6 and TNF $\alpha$, a lack of TNF receptors inhibits the recruitment of both neutrophils and macrophages in the spleen, but only neutrophils in peritoneal cavity. It seems that the migration of leukocytes is tightly controlled during noninfectious inflammation.

In conclusion, during pristane-induced non-infectious inflammation, the recruitment of dendritic cells to the spleen requires TNF $\alpha$ receptors and the presence of B cells. For neutrophil recruitment, TNF $\alpha$ and $\mathrm{LT} \alpha$ are required, in addition to $\mathrm{TNF} \alpha$ receptors and $\mathrm{B}$ cells. However, macrophage recruitment to the spleen is controlled by more factors, including IL- 6 and T cells, as well as the other regulators for neutrophil recruitment. This study may provide insight into the development of chronic inflammatory diseases associated with noninfectious stimuli.

\section{Conflicts of Interest}

The authors declare no competing interests.

\section{Authors' Contributions}

$\mathrm{Yu} \mathrm{Li}$, Junping $\mathrm{Wu}$, and Long $\mathrm{Xu}$ contributed equally to this work.

\section{Acknowledgments}

This work was supported by the National Natural Science Foundation of China (31471121, 81773394, and $81728001)$, the Natural Science Foundation of Tianjin
(17JCYBJC24700), and the Key Projects of Health and Family Planning Commission of Tianjin (16KG163 and 16KG164).

\section{References}

[1] Q. Ouyang, Z. Huang, Z. Wang, X. Chen, J. Ni, and L. Lin, "Effects of pristane alone or combined with chloroquine on macrophage activation, oxidative stress, and TH1/TH2 skewness," Journal of Immunology Research, vol. 2014, Article ID 613136, 9 pages, 2014.

[2] M. Potter and R. C. Maccardle, "Histology of developing plasma cell neoplasia induced by mineral oil in BALB/c mice," Journal of the National Cancer Institute, vol. 33, pp. 497-515, 1964.

[3] H. Chen, D. Liao, D. Cain et al., "Distinct granuloma responses in $\mathrm{C} 57 \mathrm{BL} / 6 \mathrm{~J}$ and $\mathrm{BALB} / \mathrm{cByJ}$ mice in response to pristane," International Journal of Experimental Pathology, vol. 91, pp. 460-471, 2010.

[4] M. Potter and J. S. Wax, "Genetics of susceptibility to pristaneinduced plasmacytomas in BALB/cAn: reduced susceptibility in BALB/cJ with a brief description of pristane-induced arthritis," Journal of Immunology, vol. 127, pp. 1591-1595, 1981.

[5] M. Satoh and W. H. Reeves, "Induction of lupus-associated autoantibodies in $\mathrm{BALB} / \mathrm{c}$ mice by intraperitoneal injection of pristane," The Journal of Experimental Medicine, vol. 180, pp. 2341-2346, 1994.

[6] P. Y. Lee, Y. Li, Y. Kumagai et al., "Type I interferon modulates monocyte recruitment and maturation in chronic inflammation," The American Journal of Pathology, vol. 175, pp. 2023 2033, 2009.

[7] H. Ma, S. Wan, and C. Q. Xia, "Immunosuppressive $\mathrm{CD}_{11 \mathrm{~b}^{+} \text {Ly6 }} \mathrm{C}^{\text {hi }}$ monocytes in pristane-induced lupus mouse model," Journal of Leukocyte Biology, vol. 99, pp. 1121-1129, 2016.

[8] S. Han, H. Zhuang, S. Shumyak et al., "A novel subset of antiinflammatory CD $138^{+}$macrophages is deficient in mice with experimental lupus," Journal of Immunology, vol. 199, no. 4, pp. 1261-1274, 2017.

[9] Q. Wu, X. Sun, R. Chi et al., "ROR $\gamma t$ modulates macrophage recruitment during a hydrocarbon oil-induced inflammation," PLoS One, vol. 8, article e79497, no. 11, 2013.

[10] H. Chen, D. Liao, T. M. Holl, P. Snowden, Y. Ueda, and G. Kelsoe, "Genetic regulation of pristane-induced oil granuloma responses," International Journal of Experimental Pathology, vol. 91, pp. 472-483, 2010.

[11] C. J. Ciesielski, E. Andreakos, B. M. Foxwell, and M. Feldmann, "TNF $\alpha$-induced macrophage chemokine secretion is more dependent on NF- $\kappa \mathrm{B}$ expression than lipopolysaccharidesinduced macrophage chemokine secretion," European Journal of Immunology, vol. 32, pp. 2037-2045, 2002.

[12] A. L. Kovalchuk, J. S. Kim, S. S. Park et al., "IL-6 transgenic mouse model for extraosseous plasmacytoma," Proceedings of the National Academy of Sciences of the United States of America, vol. 99, pp. 1509-1514, 2002.

[13] V. N. Ngo, H. Korner, M. D. Gunn et al., "Lymphotoxin $\alpha / \beta$ and tumor necrosis factor are required for stromal cell expression of homing chemokines in $\mathrm{B}$ and $\mathrm{T}$ cell areas of the spleen," The Journal of Experimental Medicine, vol. 189, pp. 403-412, 1999. 
[14] C. L. Zindl, T. H. Kim, M. Zeng et al., "The lymphotoxin LT $\alpha_{1} \beta_{2}$ controls postnatal and adult spleen marginal sinus vascular structure and function," Immunity, vol. 30, pp. 408420, 2009.

[15] C. Moussion and J. P. Girard, "Dendritic cells control lymphocyte entry to lymph nodes through high endothelial venules," Nature, vol. 479, pp. 542-546, 2011.

[16] M. Pasparakis, L. Alexopoulou, V. Episkopou, and G. Kollias, "Immune and inflammatory responses in TNF alphadeficient mice: a critical requirement for TNF alpha in the formation of primary B cell follicles, follicular dendritic cell networks and germinal centers, and in the maturation of the humoral immune response," The Journal of Experimental Medicine, vol. 184, pp. 1397-1411, 1996.

[17] R. Espin-Palazon, D. L. Stachura, C. A. Campbell et al., "Proinflammatory signaling regulates hematopoietic stem cell emergence," Cell, vol. 159, pp. 1070-1085, 2014.

[18] M. D. Turner, B. Nedjai, T. Hurst, and D. J. Pennington, "Cytokines and chemokines: at the crossroads of cell signalling and inflammatory disease," Biochimica et Biophysica Acta (BBA) - Molecular Cell Research, vol. 1843, pp. 2563-2582, 2014.

[19] D. Kitamura, J. Roes, R. Kuhn, and K. Rajewsky, “A B celldeficient mouse by targeted disruption of the membrane exon of the immunoglobulin $\mu$ chain gene," Nature, vol. 350, pp. 423-426, 1991.

[20] S. Fillatreau, C. H. Sweenie, M. J. McGeachy, D. Gray, and S. M. Anderton, "B cells regulate autoimmunity by provision of IL-10,” Nature Immunology, vol. 3, pp. 944-950, 2002.

[21] J. L. Croxford, S. Miyake, Y. Y. Huang, M. Shimamura, and T. Yamamura, "Invariant $\mathrm{V}_{\alpha}$ 19i T cells regulate autoimmune inflammation," Nature Immunology, vol. 7, pp. 987-994, 2006.

[22] A. Mizoguchi, E. Mizoguchi, and A. K. Bhan, "The critical role of interleukin 4 but not interferon gamma in the pathogenesis of colitis in T-cell receptor $\alpha$ mutant mice," Gastroenterology, vol. 116, pp. 320-326, 1999.

[23] K. F. Hoffmann, A. W. Cheever, and T. A. Wynn, "IL-10 and the dangers of immune polarization: excessive type 1 and type 2 cytokine responses induce distinct forms of lethal immunopathology in murine schistosomiasis," Journal of Immunology, vol. 164, pp. 6406-6416, 2000.

[24] L. M. Stasiuk, M. Ghoraishian, C. J. Elson, and S. J. Thompson, "Pristane-induced arthritis is CD4 ${ }^{+}$T-cell dependent," Immunology, vol. 90, pp. 81-86, 1997.

[25] P. F. Piguet, G. E. Grau, C. Vesin, H. Loetscher, R. Gentz, and W. Lesslauer, "Evolution of collagen arthritis in mice is arrested by treatment with anti-tumour necrosis factor (TNF) antibody or a recombinant soluble TNF receptor," Immunology, vol. 77, pp. 510-514, 1992.

[26] D. Kontoyiannis, M. Pasparakis, T. T. Pizarro, F. Cominelli, and G. Kollias, "Impaired on/off regulation of TNF biosynthesis in mice lacking TNF AU-rich elements: implications for joint and gut-associated immunopathologies," Immunity, vol. 10, pp. 387-398, 1999.

[27] J. H. Fritz, O. L. Rojas, N. Simard et al., "Acquisition of a multifunctional $\operatorname{IgA}^{+}$plasma cell phenotype in the gut," Nature, vol. 481, pp. 199-203, 2012.
[28] K. Kabashima, T. A. Banks, K. M. Ansel, T. T. Lu, C. F. Ware, and J. G. Cyster, "Intrinsic lymphotoxin- $\beta$ receptor requirement for homeostasis of lymphoid tissue dendritic cells," Immunity, vol. 22, pp. 439-450, 2005.

[29] H. S. Kang, S. E. Blink, R. K. Chin et al., "Lymphotoxin is required for maintaining physiological levels of serum IgE that minimizes Th1-mediated airway inflammation," The Journal of Experimental Medicine, vol. 198, pp. 1643-1652, 2003. 


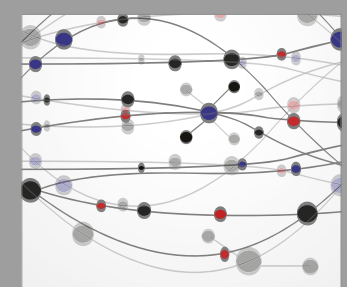

The Scientific World Journal


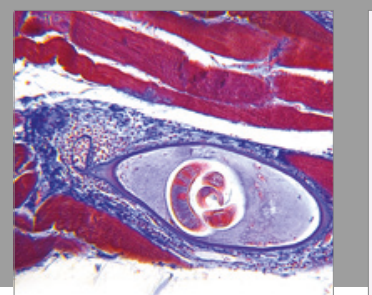

Gastroenterology Research and Practice
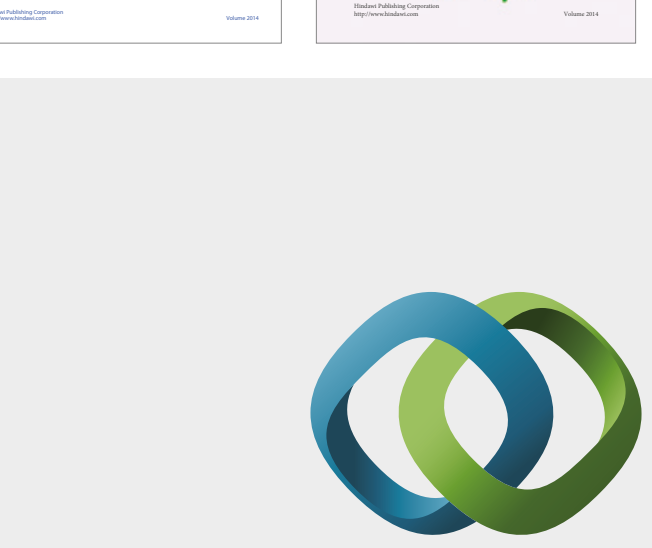

\section{Hindawi}

Submit your manuscripts at

https://www.hindawi.com
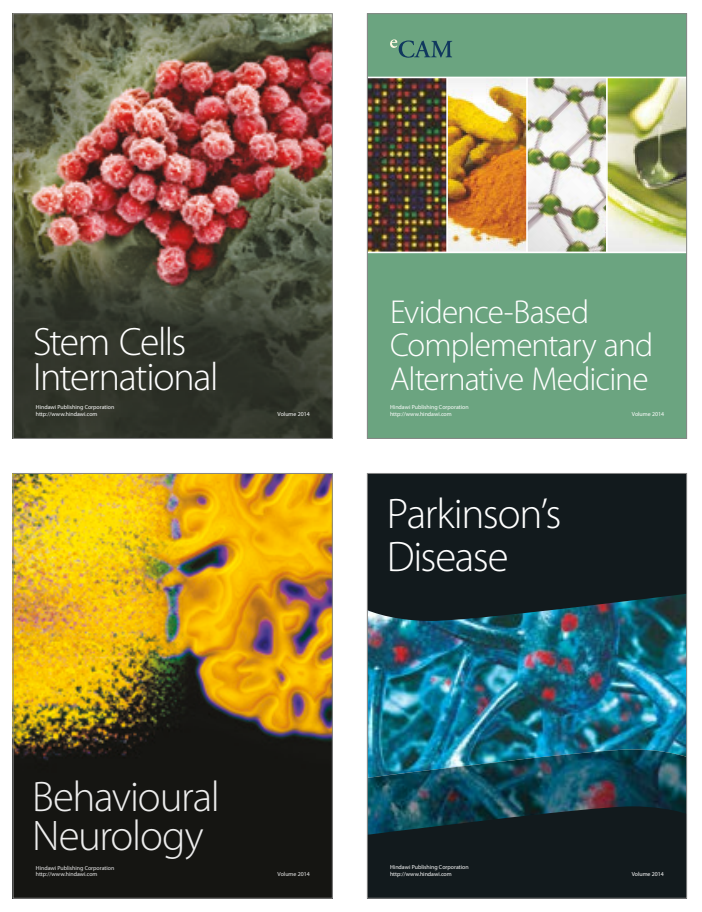
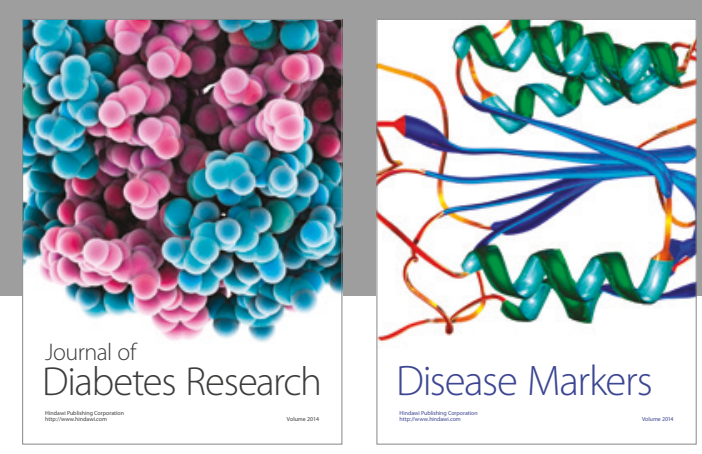

Disease Markers
